\title{
Molecular theory of ion solvation dynamics in water, acetonitrile and methanol: $A$ unified microscopic description of collective dynamics in dipolar liquids
}

\author{
SRABANI ROY and BIMAN BAGCHI ${ }^{\dagger}$ \\ Solid State and Structural Chemistry Unit, Indian Institute of Science, Bangalore 560012, \\ India \\ 'Also at the Jawaharlal Nehru Centre for Advanced Scientific Research, Bangalore 560012, \\ India
}

MS received 13 July 1993

\begin{abstract}
A recently developed microscopic theory of solvation dynamics in real dipolar liquids is used to calculate, for the first time, the solvation time correlation function in liquid acetonitrile, water and methanol. The calculated results are in excellent agreement with known experimental and computer simulation studies.
\end{abstract}

Keywords. Ion solvation dynamics; solvation time correlation function; dipolar liquids; microscopic theory of solvation dynamics.

\section{Introduction}

Our understanding of the solvation dynamics of charged species in dipolar liquids has undergone a renaissance in recent years (Bagchi 1989; Barbara and Jarzeba 1990; Maroncelli et al 1993; Roy and Bagchi 1993). Experiments (Rosenthal et al 1991) and computer simulations (Maroncelli and Fleming 1988) have shown that an ultrafast Gaussian component dominates the relaxation in both liquid acetonitrile and water. This ultrafast component seems to originate from the fast macroscopic relaxation mode of the solvent (Roy and Bagchi 1993a). In contrast, experimental (Kahlow et al 1989; Maroncelli et al 1993) and computer simulation (Fonseca and Ladanyi 1991; Maroncelli et al 1993) studies reveal methanol to be a rather different solvent, in not only being much slower, but also being nonlinear in its response to the sudden creation of an electrical charge in it. In this paper, a theoretical study of the solvation dynamics of a rigid ion in these three liquids is presented. Excellent agreement is obtained between theoretical prediction and the available experimental and simulation results in each case. Furthermore, the theory transparently explains the basic difference in behaviour between these structurally similar solvents.

\footnotetext{
* For correspondence
} 


\section{Theoretical formulation}

Within the linear-response assumption, the time-dependent solvation energy, $E_{\mathrm{sol}}(t)$, is given exactly by the following expression (Roy and Bagchi 1993a)

$$
E_{\mathrm{sol}}(t) \propto L^{-1} \int_{0}^{x} \mathrm{~d} k\left[\frac{\sin k r_{c}}{k r_{c}}\right]^{2} \cdot \frac{1}{z} \cdot\left[\frac{1}{\varepsilon(k, z)}-\frac{1}{\varepsilon(k)}\right],
$$

where $L^{-1}$ denotes an inverse Laplace transformation with respect to the Laplace frequency, $z ; r_{c}$ is the distance of closest approach between the ion and a solvent molecule; $\varepsilon(k, z)$ is the longitudinal dielectric function of the solvent, dependent on the wavevector $(k)$ and frequency $(z)$, and is related to the wavevector-dependent dielectric function $\varepsilon(k)$ by

$$
1-\frac{1}{\varepsilon(k, z)}=\left[1-\frac{1}{\varepsilon(k)}\right]\left[1-\frac{z}{z+\Sigma(k, z)}\right],
$$

with the generalized rate $\Sigma(k, z)$ given by

$$
\Sigma(k, z)=\frac{2 f_{L}(k)}{\tau_{I}^{2}\left[z+\Gamma_{R}(k, z)\right]}+\frac{p k^{2} f_{L}(k)}{\tau_{I}^{2}\left[z+\Gamma_{T}(k, z)\right]} .
$$

Here, $f_{L}(k)=1-\left(\rho_{0} / 4 \pi\right) c(110, k)$ is a measure of the static structural correlation of the pure solvent as $c(l l m, k)$ denotes the $(l l m)$ component of the direct correlation function in the intermolecular frame with $k$ parallel to the $z$ axis (Bagchi and Chandra 1991). $\rho_{0}$ is the average number density of the liquid. Note that $f_{L}(k)$ is related to $\varepsilon(k)$ by the following simple relation (Bagchi and Chandra 1991)

$$
1-(1 / \varepsilon(k))=3 Y / f_{L}(k)
$$

where the polarity parameter $3 Y=(4 \pi / 3) \beta \mu^{2} \rho_{0}$ with $\mu$ as the dipole moment of the solvent molecules. $\beta=\left(k_{B} T\right)^{-1}, k_{B}$ is the Boltzmann constant and $T$ the absolute temperature. For a solvent molecule of effective moment of inertia $I, \tau_{I}=\left(I / k_{B} T\right)^{1 / 2}$ denotes the time constant for the free inertial motion of the solvent molecules. The natural dynamics of the liquid determines the rotational and translational dissipative kernels, $\Gamma_{R}(k, z)$ and $\Gamma_{T}(k, z)$.

It is clear from the above discussion that the intermolecular orientational (in this case, dipolar) correlation function and the dissipative kernels are the two crucial ingredients of the present theory. Fortunately, recent developments in the equilibrium theory of molecular liquids have made accurate calculation of the two particle orientational correlation functions possible (Raineri et al 1992). It is shown elsewhere (Roy and Bagchi 1993) that the rotational kernel can be obtained directly from dielectric and Kerr relaxation experiments by assuming that $\Gamma_{R}(k, z)$ can be replaced by its $k=0$ limiting value. This is an inversion procedure where a microscopic relation between a correlation function and the relevant experimental observable is used to determine the dissipative kernel. For example, this procedure provides the following elegant relation with the dielectric relaxation, $\varepsilon(z)$ of the liquid (Roy and Bagchi 1993a)

$$
\frac{1}{z+\Gamma_{R}(k=0, z)}=\frac{z \tau_{I}{ }^{2}}{2 f_{L}(k)} \cdot \frac{\varepsilon_{0}\left[\varepsilon(z)-n^{2}\right]}{n^{2}\left[\varepsilon_{0}-\varepsilon(z)\right]} .
$$


In the above equation, $\varepsilon_{0}$ and $n^{2}$ are the static and the optical dielectric constants of the pure solvent. Note that all the quantities appearing on the right-hand side of (5) are experimentally measurable. Similarly, for Kerr relaxation, the dissipative kernel is given by (Roy and Bagchi 1993b)

$$
\frac{1}{z+\Gamma_{R}(k=0, z)}=\frac{\tau_{J}^{2}\left[1-z C_{20}(z)\right]}{6 f_{220}(k=0) C_{20}(z)},
$$

where $C_{20}(z)$ is the Laplace transform of the correlation function $C_{20}(t)$ usually measured in the Kerr relaxation. $f_{220}(z)=1-\left(\rho_{0} / 4 \pi\right) c(220, k)$ and can be equated to 1 for spherical and nearly spherical molecules.

The translational dissipative kernel $\Gamma_{\Upsilon}(k, z)$ is important only at intermediate to large $k$ values where it can be approximated well by its single particle value, $\zeta_{T}(z)$. In liquid water, $\zeta_{T}(z)$ is calculated using its well-known memory function relation with the Laplace transform of the velocity time auto-correlation function. The latter is obtained from the simulations of Stillinger and Rahman (1971). For acetonitrile, the translational modes are found to be unimportant except at long time durations on account of its very fast rotational motion. Similar is the situation for methanol where the motion of the molecules is rather slow. In these solvents, we may approximate the frequency-dependent friction by its zero frequency value. It is then calculated using the known values of the translational diffusion coefficients via the Stokes-Einstein relation.

The experimental results of solvation dynamics is usually expressed in terms of the solvation time correlation function $S(t)$ defined as

$$
S(t)=\frac{E_{\text {sol }}(t)-E_{\text {sol }}(\infty)}{E_{\text {sol }}(0)-E_{\text {sol }}(\infty)}
$$

The numerical results for all the three systems are presented next.

\section{Numerical results}

\subsection{Lquid acetonitrile}

Both the optical Kerr and the dielectric relaxation data have been used to calculate the rotational dissipative kernel. The normalized correlation function $C_{20}(t)$ was obtained using the Brownian oscillator representation of Cho et al (1992). The dielectric data (Barthel and Buchner 1991) consists of a Debye relaxation with $z=i \omega$ ( $\omega$ is the Fourier frequency). The translational parameter $p=0.07$. Since the dye molecule LDS-750 was used in the experimental study, we assume that the solute ion is a sphere of radius three times that of acetonitrile.

In figure 1 the theoretical and the experimental results are compared. The agreement is excellent for the case where the dissipative kernel is obtained from the Kerr relaxation, but is not satisfactory when it is calculated from the dielectric relaxation data. This is in accordance with the understanding that conventional dielectric relaxation measurements not only miss the contribution of the high frequency librational modes, but on extrapolation also lead to an underestimation of the underdamped dynamics. We have also compared the theoretical predictions with the 


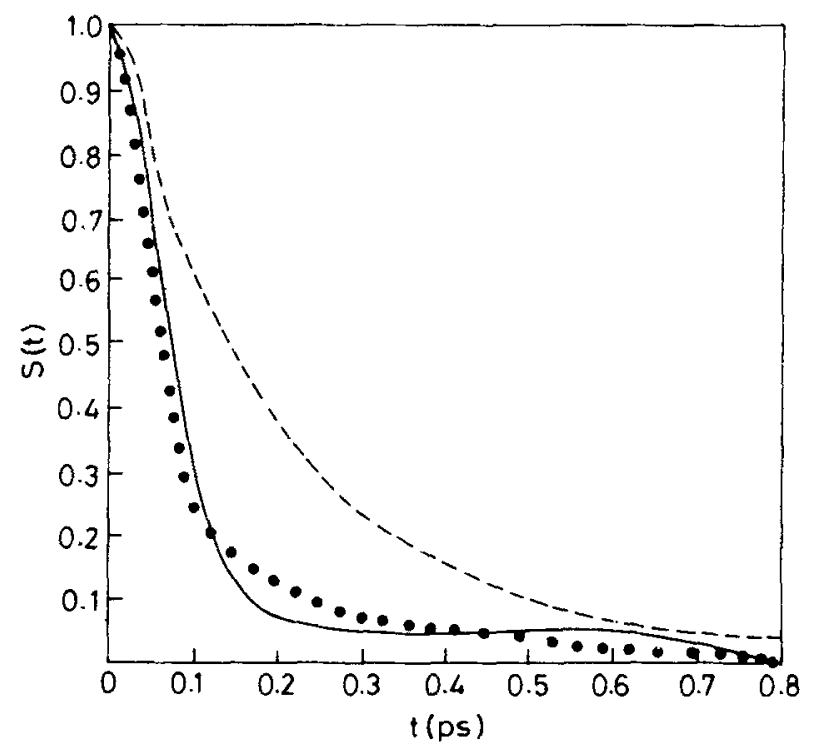

Figure 1. Comparison of the calculated solvation time correlation function, $S(t)$, with the experimental one for the solvation of LDS-750 in liquid acetonitrile. The solid and the dashed lines are the $S(t)$ calculated from the Kerr and the dielectric relaxations, respectively. The experimental result is shown by filled circles. The radius of the solute ion is three times that of an acetonitrile molecule.

computer simulations of Maroncelli (1991) where the solvent molecules are assumed to be non-polarizable. Again excellent agreement is obtained.

\subsection{Water}

For water, the correlation function, $c_{110}(k)$, and hence the $f_{110}(k)$, were calculated accurately by using the results of Raineri et al (1992). The experimental FIR absorption spectrum of water (Hasted et al 1985) shows a peak at $199 \mathrm{~cm}^{-1}$ which is believed to be mostly responsible for the high frequency dielectric relaxation. We used the following expression for $\varepsilon(z)$

$$
\varepsilon(z)=n^{2}+\frac{\varepsilon_{0}-\varepsilon_{\infty}}{1+z \tau_{D}}+\left(\varepsilon_{\infty}-n^{2}\right)\left[1-\Phi_{l i b}(z)\right],
$$

with $\tau_{D}=9.33 \mathrm{ps}, \varepsilon_{\infty}=4.86$ and $n=1.33$ (Hasted et al 1985). $\Phi_{l i b}$ is the librational moment correlation function. It is calculated using the damped oscillator model with the oscillation frequency equal to $199 \mathrm{~cm}^{-1}$ and the damping constant equal to $100 \mathrm{~cm}^{-1}$ (Hasted et al 1985). The translational parameter $p=0.02$. As shown in figure 2 , the calculated dynamics has a rich structure. The initial decay is Gaussian followed by exponential-like decay in the long time, with an oscillation in the intermediate time domain. This result, including the oscillation, is in good agreement with the computer simulations of Maroncelli and Fleming (1988). The initial sharp Gaussian decay is primarily due to the librational mode whose contribution to solvation gets greatly enhanced (compared to dielectric relaxation) due to the large value of $f_{110}(k=0)$. The initial decay can be fitted to a Gaussian form with a time constant 


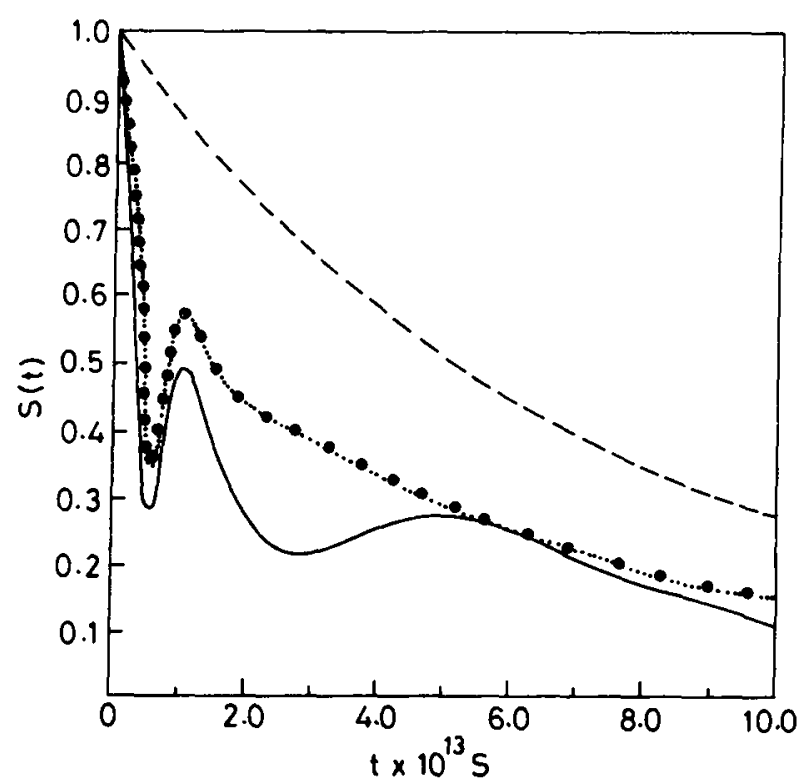

Figure 2. Calculated solvation time correlation function, $S(t)$, as a function of time for the solvation of a spherical ion in liquid water. The solid line includes both the rotational and the translational librations and the dotted line only the rotational libration. The dashed line shows the decay when only the Debye relaxation is accounted for. The solute to solvent size ratio is assumed to be $2 \cdot 25$.

$\left(\tau_{G}\right)$ equal to 42 fs and $\tau_{G}=0.42 \tau_{I}$. This implies that the solvation is much faster than the free inertial decay. The slow exponential-like decay after the oscillation has two components with time constants approximately equal to 280 and $800 \mathrm{fs}$. These are very close to the two components observed by Barbara and Jarzeba (1990a) who, however, missed the ultra-fast Gaussian component because of limited time resolution. The slow 800 is decay component is found to be due to the strong orientational correlations present at molecular length scales where $c_{110}(k)$ is positive and maximum so that $f_{110}(k)$ is small. This is essentially the orientational de Gennes narrowing. The translational librational modes of water play an important role in this regime.

\subsection{Liquid methanol}

Since experimental results are not yet fully available for liquid methanol, we compare our results with the simulations (Maroncelli et al 1993). As the molecules are not polarizable in the simulated system, the polarizability is ignored by setting the optical frequency equal to 1 and the $3 Y$ parameter equal to $4 \cdot 3276$. Here, $p=0 \cdot 267$. The results are presented in figure 3 where the simulation results of Maroncelli et al (1993) are also shown for comparison. The solvation dynamics is found to be rather similar when $\Gamma_{R}(z)$ is obtained either from the dielectric (Guillot et al 1990) or from the Kerr relaxation (Cho et al 1993). In both the cases, the theoretical predictions for the initial decay are in perfect agreement with the simulated one. The dielectric model of the dissipative kernel provides slightly better agreement in the long time.

A comparison of the relaxation pattern obtained here with those for water and acetonitrile reveals two interesting features. First, both the fast (Gaussian) and the 


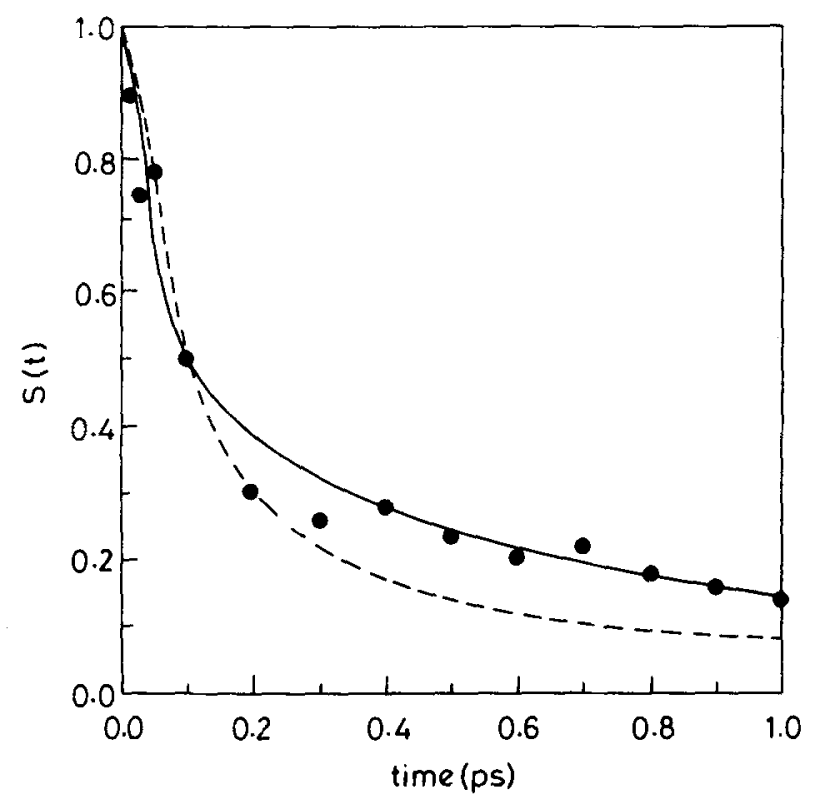

Figure 3. Solvation time correlation function, $S(t)$, calculated from the dielectric relaxation (solid line) and the Kerr relaxation (dashed line) for the solvation of a rigid ion in liquid methanol. The simulation results are shown by the solid circles. The solute is of the same size as coumarin.

slow (exponential-like) components are much slower in methanol than in the other two solvents. Second, the Gaussian component carries a smaller weight in methanol. The available experimental studies (Kahlow et al 1989; Maroncelli et al 1993) for solvation dynamics in methanol suffer from a limited temporal resolution. Comparison between theoretical calculations (now performed with polarizability effects included which makes solvation even faster) with experimental results suggests that the experiments have missed almost all of the fast Gaussian component that contributes about $40-50 \%$ of the total energy relaxation. Thus, we conclude that the available experiments have detected only the last $50 \%$ of the decay. This is also in agreement with the conclusion of Maroncelli et al (1993).

\section{Conclusion}

In this communication microscopic calculations of ion solvation dynamics in three common dipolar liquids are presented. The theory is sufficiently simple so that a detailed numerical calculation of the solvation time correlation function can be carried out for the common dipolar liquids. The agreement between theory and available experimental and computer simulation results is excellent in each case. It is found that while the librational modes and dipolar interaction induced effects are important in water, it is the single particle rotation that drives the ultrafast solvation in acetonitrile. Methanol exhibits markedly different dynamics because here the orientational motion is slow and the energy relaxation derives a significant contribution from the solvent translational modes. These factors also make the response of methonal nonlinear, as explained in more detail elsewhere (Roy et al 1993). 


\section{References}

Bagchi B 1989 Annu. Rev. Phys. Chem 40115

Bagchi B and Chandra A 1991 Adv. Chem. Phys. 801

Barbara P F and Jarzeba W 1990a Acc.Chem. Res. 21195

Barbara P F and Jarzeba W 1990b Adv. Photochem. 151

Barthel J and Buchner R 1991 Pure Appl. Chem. 631473

Cho M, Du M, Scherer N F, Fleming G R and Mukamel S $1993 \mathrm{~J}$. Chem. Phys. (submitted)

Cho M, Rosenthal S J, Scherer N F, Ziegler L D and Fleming G R 1992 J. Chem. Phys. 965033

Fonseca T and Ladanyi B M $1991 \mathrm{~J}$. Chem. Phys. 952116

Guillot B, Marteau P and Obriot J 1990 J. Chem. Phys. 936148

Hasted J B, Hussain S K, Frescura F M and Birch J R 1985 Chem. Phys. Lett. 118622

Kahlow M A, Jarzeba W, Kang T J and Barbara P F 1989 J. Chem. Phys. 90151

Maroncelli M $1991 \mathrm{~J}$. Chem. Phys. 942084

Maroncelli M and Fleming G R 1988 J. Chem. Phys. 895044

Maroncelli M, Kumar P V, Papazyan A, Horng M L, Rosenthal S and Fleming G R 1993 Studies of the inertial component of polar solvation. In Ultrafast reaction dynamics and solvent effects (eds.) Y Gauduel and P J Rossky (New York: American Institute of Physics)

Raineri F O, Resat H and Freidman H L 1992 J. Chem. Phys. 963058

Rosenthal S J, Xie X, Du M and Fleming G R 1991 J. Chem. Phys. 954715

Roy S and Bagchi B 1993a J. Chem. Phys. 991310

Roy S and Bagchi B 1993b J. Chem. Phys. (submitted)

Roy S, Komath S S and Bagchi B $1993 \mathrm{~J}$. Chem. Phys. (in press)

Stillinger F H and Rahman A 1971 J. Chem. Phys. 601545 